\title{
The Diversity of Chrysophycean Algae in an Arctic Zone of River and Sea Water Mixing, Russia
}

\author{
Alena D. Firsova ${ }^{1}$, Anna Yu Bessudova1 ${ }^{*}$, Larisa M. Sorokovikova², Irina V. Tomberg'2, \\ Yelena V. Likhoshway ${ }^{1}$ \\ ${ }^{1}$ Department of Cell Ultrastructure, Limnological Institute, Siberian Branch, Russian Academy of Sciences, \\ Irkutsk, Russia \\ ${ }^{2}$ Laboratory of Hydrochemistry and Atmosphere Chemistry, Limnological Institute, Siberian Branch, Russian \\ Academy of Sciences, Irkutsk, Russia \\ Email: firsova@lin.irk.ru, ̌annabessudova@mail.ru, lara@lin.irk.ru, kaktus@lin.irk.ru, yel@lin.irk.ru
}

Received 10 July 2015; accepted 27 September 2015; published 30 September 2015

Copyright (C) 2015 by authors and Scientific Research Publishing Inc.

This work is licensed under the Creative Commons Attribution International License (CC BY). http://creativecommons.org/licenses/by/4.0/

(c) (i) Open Access

\begin{abstract}
According to the results of studies in the region of lower Yenisei River to the Kara Sea shelf in September 2009, the flora of chrysophyte algae in the zone of river and sea water mixing comprises 43 species of the classes Chrysophyceae and Synurophyceae. Most of them are cosmopolitan and widespread, but four rare species have also been recorded. The most frequent species are Synura petersenii f. petersenii, S. petersenii f. kufferathii, Spiniferomonas takahashii, Mallomonas acaroides, and M. crassisquama. The composition of chrysophyte flora in the study region is similar to that in some lakes of the Taimyr Peninsula. It has been found that the diversity of chrysophytes in the zone of river and sea water mixing depends on water salinity, with a group of species occurring at increased salinity levels. It includes Chrysosphaerella coronacircumspina (5.2\%), Kephyrion spirale (5.2\%) and Mallomonas crassisquama (8\%o). These data contribute to knowledge of how the distribution of chrysophytes responds to changes in ecological conditions such as water salinity, turbidity, $\mathrm{pH}$, and concentrations of oxygen and mineral phosphorus.
\end{abstract}

\section{Keywords}

Chrysophycean Algae, Salinity, Arctic Zone

\footnotetext{
${ }^{*}$ Corresponding author.
} 


\section{Introduction}

Chrysophyte algae (Chrysophyta) are an important component of phytoplankton that is highly responsive to changes in the aquatic environment [1]. They mainly inhabit cold, fresh waters [2]-[4], but there is evidence that many species are tolerant of high salinity levels [1] [5] [6]. In Arctic regions, species are known that occur both in fresh and salt waters, e.g. Paraphysomonas imperforata and Mallomonas tonsurata; brackish waters of Greenland are inhabited by Paraphysomonas vestita and Synura petersenii [7].

In recent years, a number of studies have been performed on water bodies of the Arctic zone, where the climate is severe with long, cold winters and a short open-water period. In the majority of lakes studies, this period is only 2 - 3 months, and the lakes are oligotrophic or even ultraoligotrophic. Chrysophytes are proved to be a dominant group of phytoplankton in many of these lakes [7]-[18], accounting for up to $60 \%-80 \%$ of its total abundance and 50\% - 70\% of total biomass [18]. The highest diversity of chrysophytes is observed in the region of Bol'shezemelskaya tundra: 95 species and infraspecific taxa, including 46 species of the genus Mallomonas and 16 species of the genus Dinobryon. The authors attribute this to favorable conditions for the development of chrysophytes in lakes of the above region: water temperature $12^{\circ} \mathrm{C}-19^{\circ} \mathrm{C}$, pH $5.5-7.5$, low specific conductance (19 - $\left.59 \mu \mathrm{S} \cdot \mathrm{cm}^{-1}\right)$, and the absence of competition with cyanobacteria [11] [13] [19]. Analyzing the taxonomic diversity of phytoplankton in oligotrophic lakes of the Polar Urals, Voloshko [12] [14] [20] [21] recorde 47 chrysophyte species, with representatives of the genus Mallomonas (20 species) also holding a leading position. The $\mathrm{pH}$ of the water during the study period is almost neutral (pH 6.7 - 7.3). In Swedish Lapland, the chrysophyte flora of lakes and pools is found to consist of 28 species from 5 genera, with the prevalence of Mallomonas (16 species) and Synura (5 species) [17]. A total of 23 chrysophyte species are recorded in fresh water bodies of the Taimyr Peninsula located between the Yenisei Gulf (the Kara Sea) and Khatanga Gulf (the Laptev Sea), which are close to the region of our study [8]. The list of chrysophytes previously found in the Khantay Reservoir on the Yenisei River, which flows into the Kara Sea, comprises 67 species, including 18 species of the genus Dinobryon Ehrenb [22].

In this study, electron microscopic methods are used to evaluate the diversity of chrysophytes depending on hydrochemical parameters in the zone of mixing of Yenisei River and Kara Sea waters.

\section{Material and Methods}

During an expedition carried out in September 1-24, 2009, samples of phytoplankton and water were taken at 33 stations located in the lower Yenisei (st. 24-34), Yenisei Gulf (st. 1-4, 10, 22, 23), Gydan Bay (st. 11-19), and the Kara Sea shelf (st. 5-9, 20, 21) (Figure 1, Table 1) within the RAS Presidium Program "Integrated Research of Arctic shelf”, project 20.7.

Water samples for chemical analysis were cleared of suspended matter by filtering through membranes with a pore size of $0.45 \mu \mathrm{m}$ (VladiSart, Russia). Nutrient elements were determined by colorimetric methods [23]; cations, by atomic absorption and flame emission spectroscopy [24]; anions, by HPLC [25]. Biogenic elements were determined by colorimetric methods [23] [24]. Nitrate nitrogen was determined by HPLC, nitrites-with Griss reagent; ammonium nitrogen - with indophenol method; total phosphorus - with potassium persulphate (burning at $150^{\circ} \mathrm{C}$ ); the determination, like with mineral phosphorus, was finished by Denigès-Atkins method. Dissolved silicic acid was determined using a yellow silica-molybdenum unit [24].

Qualitative samples of phytoplankton were collected with a Juday plankton net (nylon fabric mesh No. 70) and fixed with 70\% ethyl alcohol. Scaled chrysophytes were identified and analyzed using scanning and transmission electron microscopy (SEM and TEM). Samples for SEM were concentrated on membrane filters with a pore diameter of $1.2 \mu \mathrm{m}$ (Millipore, United States), sputter-coated with gold, and analyzed under a Philips 525 M scanning electron microscope. In case of TEM, a concentrated sample was placed onto a Formvar-coated grid and examined under a LEO 906E transmission electron microscope. A total of 66 samples were analyzed. Sørensen's coefficient of similarity was calculated as described [26] in order to determine the degree of difference with water bodies of Bol'shezemel'skaya Tundra, Taymyr Peninsula and Swedish Lapland.

Sørensen's coefficient of similarity was determined by formula:

$$
\mathrm{K}=\frac{2 c}{a+b} \times 100,
$$

where $c$ is the number of common species; $a$ is the number of species in the water bodies of ecosystems studied; 


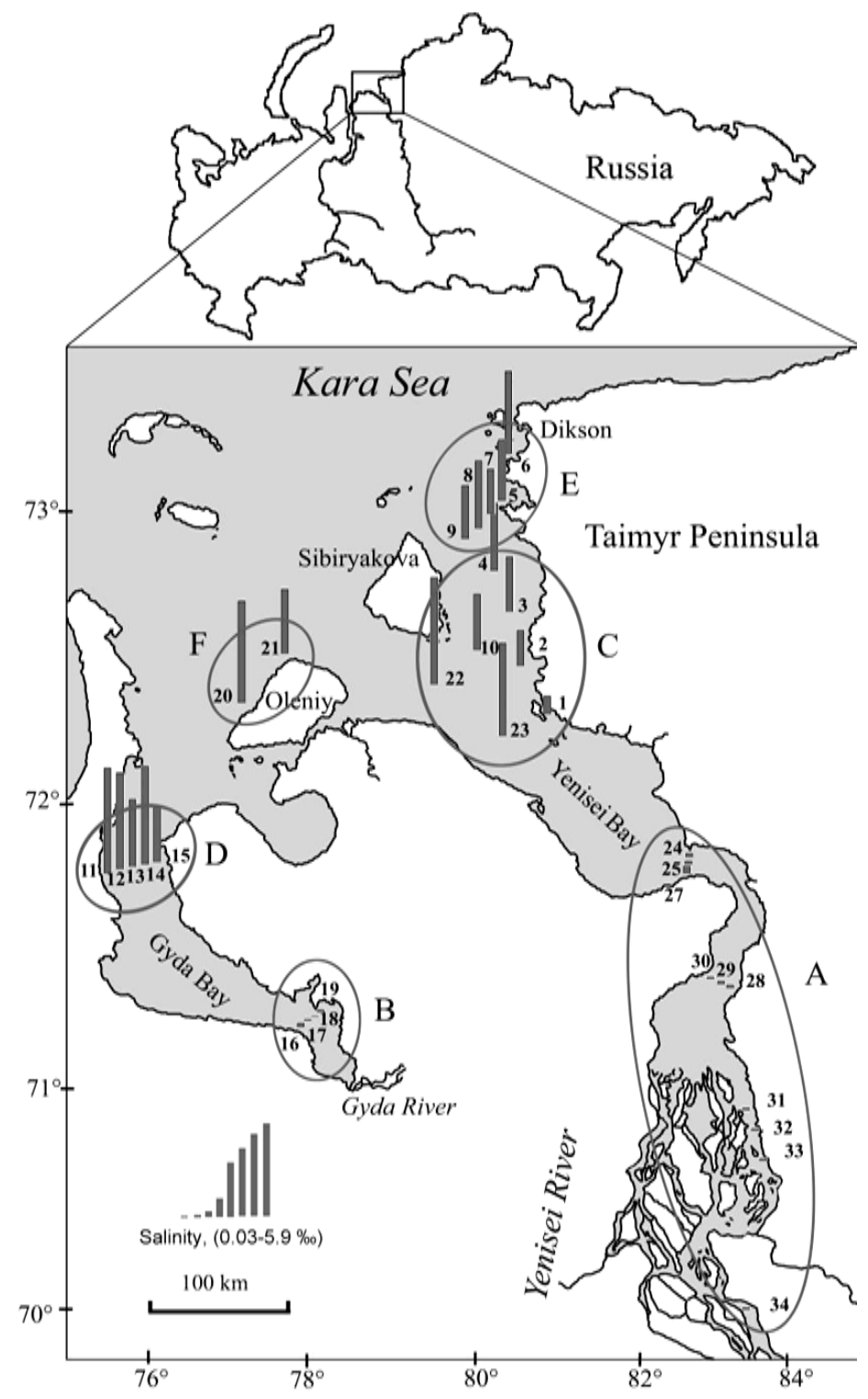

Figure 1. Schematic map of the study region and locations of sampling stations.

$b$ is the number of species in the water bodies at the compared territories.

Floral link coefficient is calculated by the formula:

$$
K=\frac{r-(x+y)}{r+(x+y)},
$$

where $x$ is the number of specific species in the water bodies of studied ecosystems; $y$ is the number of specific species in the water bodies at the compared territories; $r$ is the number of common species [26].

\section{Results}

\subsection{Characteristics of the Study Region}

Conditions of formation, hydrophysical parameters, and chemical composition of water masses in the study region are diverse (Table 1). Water depths are relatively shallow (at most 29 m), especially in Gydan Bay (2 - 11 
Table 1. Coordinates of sampling stations and some physicochemical parameters of water in the study region (2009).

\begin{tabular}{|c|c|c|c|c|c|c|c|c|c|c|c|}
\hline $\begin{array}{l}\text { Station } \\
\text { No. }\end{array}$ & Date & Coordinates & $\begin{array}{l}\text { Max. } \\
\text { depth, m }\end{array}$ & $\mathrm{T},{ }^{\circ} \mathrm{C}$ & $\mathrm{pH}$ & $\begin{array}{c}\mathrm{O}_{2}, \\
\mathrm{mg} \cdot \mathrm{l}^{-1}\end{array}$ & $\begin{array}{l}\text { Turbidity, } \\
\mathrm{mg} \cdot \mathrm{l}^{-1}\end{array}$ & $\begin{array}{l}\text { Salinity, } \\
\% \text { \% }\end{array}$ & $\begin{array}{c}\mathrm{P}, \\
\mu \mathrm{g} \cdot \mathrm{l}^{-1}\end{array}$ & $\begin{array}{l}\text { Conductivity, } \\
\mu \mathrm{S} \cdot \mathrm{cm}^{-1}\end{array}$ & $\begin{array}{l}\mathrm{Si} \\
\mathrm{mg} \cdot \mathrm{l}^{-1}\end{array}$ \\
\hline 1 & Sept. 1 & $\begin{array}{l}72^{\circ} 26^{\prime} 27.00^{\prime \prime} \mathrm{N} \\
80^{\circ} 49^{\prime} 37.20^{\prime \prime} \mathrm{E}\end{array}$ & 29 & 11.6 & 8.09 & 10.4 & 5.85 & 1 & 8 & 2000 & 3.97 \\
\hline 2 & Sept. 1 & $\begin{array}{c}72^{\circ} 37^{\prime} 58.80^{\prime \prime N}, \\
80^{\circ} 33^{\prime} 26.46^{\prime \prime} \mathrm{E}\end{array}$ & 15 & 9.9 & 8.05 & 10.7 & 9.25 & 2 & 8 & 3420 & 3.31 \\
\hline 3 & Sept. 1 & $\begin{array}{l}72^{\circ} 46^{\prime} 49.92 " \mathrm{~N} \\
80^{\circ} 26^{\prime} 16.92^{\prime \prime} \mathrm{E}\end{array}$ & 15 & 9.5 & 7.98 & 10.7 & 13.02 & 3.12 & 9 & 5540 & 3.03 \\
\hline 4 & Sept. 1 & $\begin{array}{c}73^{\circ} 00^{\prime} 45.47^{\prime \prime N} \\
80^{\circ} 16^{\prime} 15.20^{\prime \prime} \mathrm{E}\end{array}$ & 19 & 10.2 & 8.03 & 10.7 & 6.04 & 3.8 & 6 & 7150 & 2.85 \\
\hline 5 & Sept. 1 & $\begin{array}{l}73^{\circ} 10^{\prime} 43.34^{\prime \prime N}, \\
80^{\circ} 12^{\prime} 35.15^{\prime \prime}\end{array}$ & 26.5 & 9.4 & 8.06 & 10.8 & 6.98 & 3.42 & 6 & 6050 & 2.68 \\
\hline 6 & Sept. 1 & $\begin{array}{l}73^{\circ} 23^{\prime} 57.78^{\prime \prime N}, \\
80^{\circ} 28^{\prime} 23.60^{\prime \prime} \mathrm{E}\end{array}$ & 26.5 & 8.6 & 8.07 & 10.9 & 6.98 & 5 & 8 & 8540 & 2.49 \\
\hline 7 & Sept. 4 & $\begin{array}{l}73^{\circ} 09^{\prime} 00.28 " \mathrm{~N} \\
80^{\circ} 16^{\prime} 24.84^{\prime \prime} \mathrm{E}\end{array}$ & 25 & 8.2 & 8.07 & 8.7 & 6.98 & 2.5 & 10 & 5180 & 2.89 \\
\hline 8 & Sept. 4 & $\begin{array}{c}73^{\circ} 07^{\prime} 05.69^{\prime \prime N}, \\
80^{\circ} 08^{\prime} 06.48^{\prime \prime} \mathrm{E}\end{array}$ & 25 & 9.6 & 8.07 & 10.9 & 7.36 & 3.8 & 13 & 7410 & 2.79 \\
\hline 9 & Sept. 4 & $\begin{array}{c}73^{\circ} 04^{\prime} 57.23 " \mathrm{~N} \\
79^{\circ} 58^{\prime} 49.95^{\prime \prime} \mathrm{E}\end{array}$ & 23 & 9.9 & 8.11 & 10.9 & - & 3 & 12 & 6060 & 3.03 \\
\hline 10 & Sept. 5 & $\begin{array}{l}72^{\circ} 38^{\prime} 48.33^{\prime \prime N} \\
80^{\circ} 08^{\prime} 09.76 " \mathrm{E}\end{array}$ & 17 & 9.6 & 8.01 & 10.7 & 12.83 & 3.12 & 10 & 6020 & 2.67 \\
\hline 11 & Sept. 6 & $\begin{array}{l}71^{\circ} 45^{\prime} 59.98 " \mathrm{~N} \\
75^{\circ} 17^{\prime} 24.64^{\prime \prime} \mathrm{E}\end{array}$ & 4.5 & 9.9 & 7.86 & 11.0 & 33.77 & 5.9 & 14 & 10,030 & 0.92 \\
\hline 12 & Sept. 7 & $\begin{array}{l}71^{\circ} 46^{\prime} 25.60^{\prime \prime N} \\
75^{\circ} 31^{\prime} 17.23^{\prime \prime}\end{array}$ & 6.2 & 9 & 7.89 & 11.0 & 50.00 & 5.4 & 20 & 9050 & 1.21 \\
\hline 13 & Sept. 7 & $\begin{array}{l}71^{\circ} 46^{\prime} 33.34 " \mathrm{~N} \\
75^{\circ} 48^{\prime} 33.60^{\prime \prime} \mathrm{E}\end{array}$ & 10.7 & 9.1 & 7.94 & 10.8 & 54.53 & 3.8 & 23 & 7890 & 1.75 \\
\hline 14 & Sept. 7 & $\begin{array}{l}71^{\circ} 46^{\prime} 36.89 " \mathrm{~N} \\
75^{\circ} 58^{\prime} 49.61^{\prime \prime} \mathrm{E}\end{array}$ & 6.7 & 9.3 & 7.93 & 11.0 & 56.60 & 5.5 & 24 & 10,480 & 1.28 \\
\hline 15 & Sept. 7 & $\begin{array}{l}71^{\circ} 46^{\prime} 51.47^{\prime \prime} \mathrm{N} \\
76^{\circ} 04^{\prime} 14.11^{\prime \prime} \mathrm{E}\end{array}$ & 5.5 & 9.9 & 7.91 & 11.0 & 108.49 & 3.1 & 21 & 6090 & 0.92 \\
\hline 16 & Sept. 9 & $\begin{array}{l}71^{\circ} 10^{\prime} 30.63 " \mathrm{~N} \\
77^{\circ} 22^{\prime} 37.69^{\prime \prime} \mathrm{E}\end{array}$ & 2 & 9.6 & 7.65 & 11.0 & 60.00 & 0.2 & 0 & 546 & 0.14 \\
\hline 17 & Sept. 9 & $\begin{array}{l}71^{\circ} 11^{\prime} 37.40^{\prime \prime N} \\
77^{\circ} 27^{\prime} 34.01^{\prime \prime} \mathrm{E}\end{array}$ & 4 & 10.3 & 7.64 & 10.9 & 85.28 & 0.04 & 2 & 86.8 & 0.15 \\
\hline 18 & Sept. 9 & $\begin{array}{l}71^{\circ} 13^{\prime} 28.61^{\prime \prime N} \\
77^{\circ} 33^{\prime} 56.36^{\prime \prime} \mathrm{E}\end{array}$ & 7.5 & 10.4 & 7.57 & 11.1 & 71.51 & 0.03 & 1 & 42.8 & 0.11 \\
\hline 19 & Sept. 9 & $\begin{array}{l}71^{\circ} 14 ' 40.14 " \mathrm{~N} \\
77^{\circ} 41^{\prime} 09.22^{\prime \prime} \mathrm{E}\end{array}$ & 4 & 10.6 & 7.55 & 11.3 & 197.55 & 0.1 & 5 & 205 & 0.11 \\
\hline 20 & Sept. 10 & $\begin{array}{c}72^{\circ} 27^{\prime} 13.56^{\prime \prime N} \\
77^{\circ} 03^{\prime} 19.32^{\prime} \mathrm{E}\end{array}$ & 5 & 8.4 & 7.98 & 10.9 & 133.40 & 5.7 & 20 & 10,380 & 2.10 \\
\hline 21 & Sept. 10 & $\begin{array}{l}72^{\circ} 37^{\prime} 12.81 " \mathrm{~N} \\
77^{\circ} 31^{\prime} 29.63 " \mathrm{E}\end{array}$ & 5 & 8.8 & 8.01 & 10.8 & 56.60 & 3.6 & 19 & 7510 & 2.11 \\
\hline 22 & Sept. 11 & $\begin{array}{c}72^{\circ} 33^{\prime} 24.17^{\prime \prime N} \\
79^{\circ} 18^{\prime} 08.27^{\prime \prime} \mathrm{E}\end{array}$ & 13.5 & 8.1 & 8.03 & 10.9 & 14.72 & 8 & 17 & 12,730 & 2.71 \\
\hline 23 & Sept. 13 & $\begin{array}{l}72^{\circ} 20^{\prime} 46.75^{\prime \prime} \mathrm{N} \\
80^{\circ} 15^{\prime} 11.66^{\prime \prime} \mathrm{E}\end{array}$ & 10.5 & 8.4 & 7.98 & 11.0 & 128.68 & 5.2 & 26 & 9030 & 2.84 \\
\hline
\end{tabular}




\begin{tabular}{|c|c|c|c|c|c|c|c|c|c|c|c|}
\hline \multicolumn{12}{|c|}{ Continued } \\
\hline 24 & Sept. 15 & $\begin{array}{l}71^{\circ} 52^{\prime} 31.04 " \mathrm{~N} \\
82^{\circ} 49^{\prime} 22.12^{\prime \prime} \mathrm{E}\end{array}$ & 9.5 & 9.9 & 8.04 & 10.7 & 17.36 & 0.2 & 13 & 299 & 2.26 \\
\hline 25 & Sept. 15 & $\begin{array}{l}71^{\circ} 50^{\prime} 30.14 " \mathrm{~N} \\
82^{\circ} 47^{\prime} 06.19^{\prime \prime} \mathrm{E}\end{array}$ & 23.9 & 9.8 & 8.04 & 10.9 & 27.17 & 0.16 & 11 & 215 & 2.29 \\
\hline 27 & Sept. 15 & $\begin{array}{l}71^{\circ} 46^{\prime} 08.09 " \mathrm{~N} \\
82^{\circ} 42^{\prime} 23.52^{\prime \prime} \mathrm{E}\end{array}$ & 6.9 & 9.2 & 7.95 & 11.0 & 68.87 & 0.4 & 15 & 704 & 2.21 \\
\hline 28 & Sept. 16 & $\begin{array}{l}71^{\circ} 24^{\prime} 13.90^{\prime \prime} \mathrm{N} \\
83^{\circ} 23^{\prime} 05.10^{\prime \prime} \mathrm{E}\end{array}$ & 9.2 & 8.8 & 7.96 & 11.1 & 20.57 & 0.14 & 6 & 164 & 2.02 \\
\hline 29 & Sept. 16 & $\begin{array}{l}71^{\circ} 24^{\prime} 17.97^{\prime \prime N} \\
83^{\circ} 11^{\prime} 47.95^{\prime \prime}\end{array}$ & 23 & 9.5 & 7.95 & 10.8 & 14.53 & 0.14 & 8 & 158 & 2.05 \\
\hline 30 & Sept. 16 & $\begin{array}{l}71^{\circ} 24^{\prime} 04.76^{\prime \prime N} \\
83^{\circ} 03^{\prime} 58.88^{\prime \prime} \mathrm{E}\end{array}$ & 10 & 8.7 & 8.06 & - & 14.53 & 0.1 & 9 & 163 & 2.09 \\
\hline 31 & Sept. 18 & $\begin{array}{c}70^{\circ} 43^{\prime} 05.78^{\prime \prime} \mathrm{N} \\
83^{\circ} 30^{\prime} 07.66^{\prime \prime} \mathrm{E}\end{array}$ & 16.5 & 10.5 & 7.95 & 10.6 & 10.38 & 0.1 & 6 & 163 & 1.98 \\
\hline 32 & Sept. 18 & $\begin{array}{l}70^{\circ} 37^{\prime} 40.10^{\prime \prime} \mathrm{N} \\
83^{\circ} 28^{\prime} 13.79^{\prime \prime} \mathrm{E}\end{array}$ & 12.7 & 10.5 & 7.93 & - & 8.11 & 0.1 & 6 & 166.2 & 2.01 \\
\hline 33 & Sept. 18 & $\begin{array}{l}70^{\circ} 30^{\prime} 41.34 " \mathrm{~N} \\
83^{\circ} 21^{\prime} 06.43^{\prime \prime} \mathrm{E}\end{array}$ & - & 10.8 & 7.93 & - & 7.74 & 0.1 & 6 & 161.3 & 2.01 \\
\hline 34 & Sept. 24 & $\begin{array}{l}69^{\circ} 58^{\prime} 46.38^{\prime \prime N} \\
83^{\circ} 32^{\prime} 26.79^{\prime \prime} \mathrm{E}\end{array}$ & - & 10.2 & 8.2 & 11.7 & 4.34 & 0.1 & 2 & 179.3 & 2.23 \\
\hline
\end{tabular}

$\mathrm{m})$. The salinity of surface waters in the study period varied from $0.03 \%$ in the southern part of Gydan Bay to $5.7 \%$ in its northern part and in the Kara Se shelf. Water temperature in the lower Yenisei varied between $9.2^{\circ} \mathrm{C}$ and $10.8^{\circ} \mathrm{C}$, gradually decreasing to $8.2^{\circ} \mathrm{C}-8.6^{\circ} \mathrm{C}$ in the Yenisei Gulf and Kara Sea shelf. Water temperature in Gydan Bay was almost uniform throughout its area $\left(9.6^{\circ} \mathrm{C}-10.6^{\circ} \mathrm{C}\right)$. Dissolved oxygen reached a peak of 11.7 $\mathrm{mg} \cdot \mathrm{l}^{-1}$ in the lower Yenisei, varied between 10.3 and 10.9 in Gydan Bay, and decreased to $8.7 \mathrm{mg} \cdot \mathrm{l}^{-1}$ in the coastal sea waters. Water $\mathrm{pH}$ values were fairly high, from 7.55 in the lower Yenisei and Gydan Bay to 8.2 in the Yenisei Gulf. The concentration of dissolved silicon was the lowest in Gydan Bay $\left(0.10-0.15 \mathrm{mg} \cdot \mathrm{l}^{-1}\right)$, slightly varied along the river segment (from 2.0 to $2.2 \mathrm{mg} \cdot \mathrm{l}^{-1}$ ), and increased to $2.7-3.3 \mathrm{mg} \cdot \mathrm{l}^{-1}$ in the Yenisei Gulf. Minerl phosphorus concentration gradually increased from 2 to $15 \mu \mathrm{g} \cdot \mathrm{l}^{-1}$ along the river segment and varied between 5 and $13 \mu \mathrm{g} \cdot \mathrm{l}^{-1}$ in water areas of Yenisei Gulf and Gydan Bay.

\subsection{Species Diversity}

As noted previously [26], the composition of phytoplankton in the lower Yenisei River, Yenisei Gulf, Gydan Bay, and Kara Sea shelf was dominated by diatoms, which accounted for $40 \%$ to $90 \%$ of the total species number. The diversity of planktonic chrysophytes was significantly lower, and their contribution to the species list was no more than $10 \%$ - 25\% [26]. In this study, we recorded 43 chrysophyte species and infraspecific taxa. These were mainly widespread and cosmopolitan representatives of the genera Mallomonas (13 species), Spiniferomonas (6), Dinobryon (5), Synura (4), Kephyrion (5), Paraphysomonas (3), Stenokalyx (1), Chrysosphaerella (3), and Pseudokephyrion (1) (Table 1).

\subsection{Fresh Waters}

In the lower Yenisei (st. 24-34), chrysophytes were represented by 27 species. The group of often found species included Spiniferomonas takahashii, S. trioralis, Paraphysomonas gladiata, P. vestita, Synura petersenii, Mallomonas acaroides and M. crassisquama were also often found at st. 1 and 25 (Table 2, Figure 2, Figure 3). Dinobryon sociale and species of the genera Pseudokephyrion and Kephyrion were common components of plankton samples, as was previously described for other Arctic water bodies [18] [21].

The chrysophyte community in the freshwater part of Gydan Bay (st. 11-19) consisted of 25 species, mainly cosmopolitan and widespread. The most frequent species were Spiniferomonas serrata, Mallomonas crassisquama, Synura petersenii, and Chrysosphaerella brevispina. The genus Spiniferomonas was better represented 
Table 2. Species composition of chrysophytes in the lower Yenisei River, Yenisei Gulf, Gydan Bay, and coastal waters of the Kara Sea.

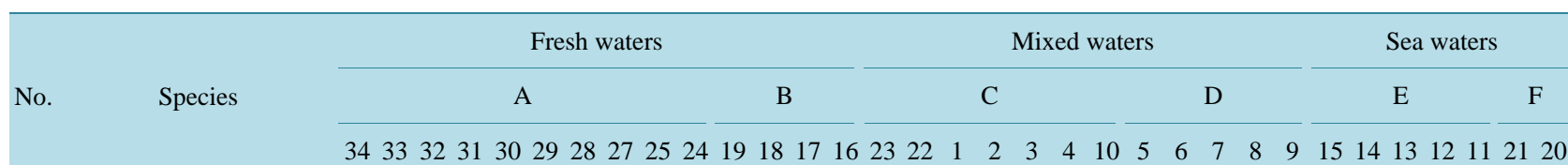

\section{Chrysosphaerella} brevispina Korschikov

2 C. coronacircumspina Wujek and Kristiansen

3 Kephyrion amphorula Conrad

$4 \quad$ K. boreale Skuja

$5 \quad$ K. inconstans (Schmid) Bourrelly

6 K. spirale (Lackey) Conrad

\section{Mallomonas acaroides} Perty em. Ivanov

M. alpina Pascher et

8 Ruttner em. Asmund et Kristiansen

$9 \quad \begin{gathered}\text { M. akrokomos } \\ \text { Ruttner in Pascher }\end{gathered}$

M. caudata Ivanov em. Krieger

\section{M. costata} Dürrschmidt

\section{M. crassisquama} (Asmund) Fott

\section{M. elongata Perty}

M. multiunca var. pocosinensis Siver

\section{M. punctifera} Korschikov

\section{M. striata var. striata} Asmund

M. tonsurata Telling em. Krieger

\section{Pseudokephyrion} entzii Conrad

\section{P. undulatissimum} Scherffel

Chrysococcus furcatus (Dolgoff) Nicholls

Dinobryon bavaricum Imhof

D. cylindricum Imhof 


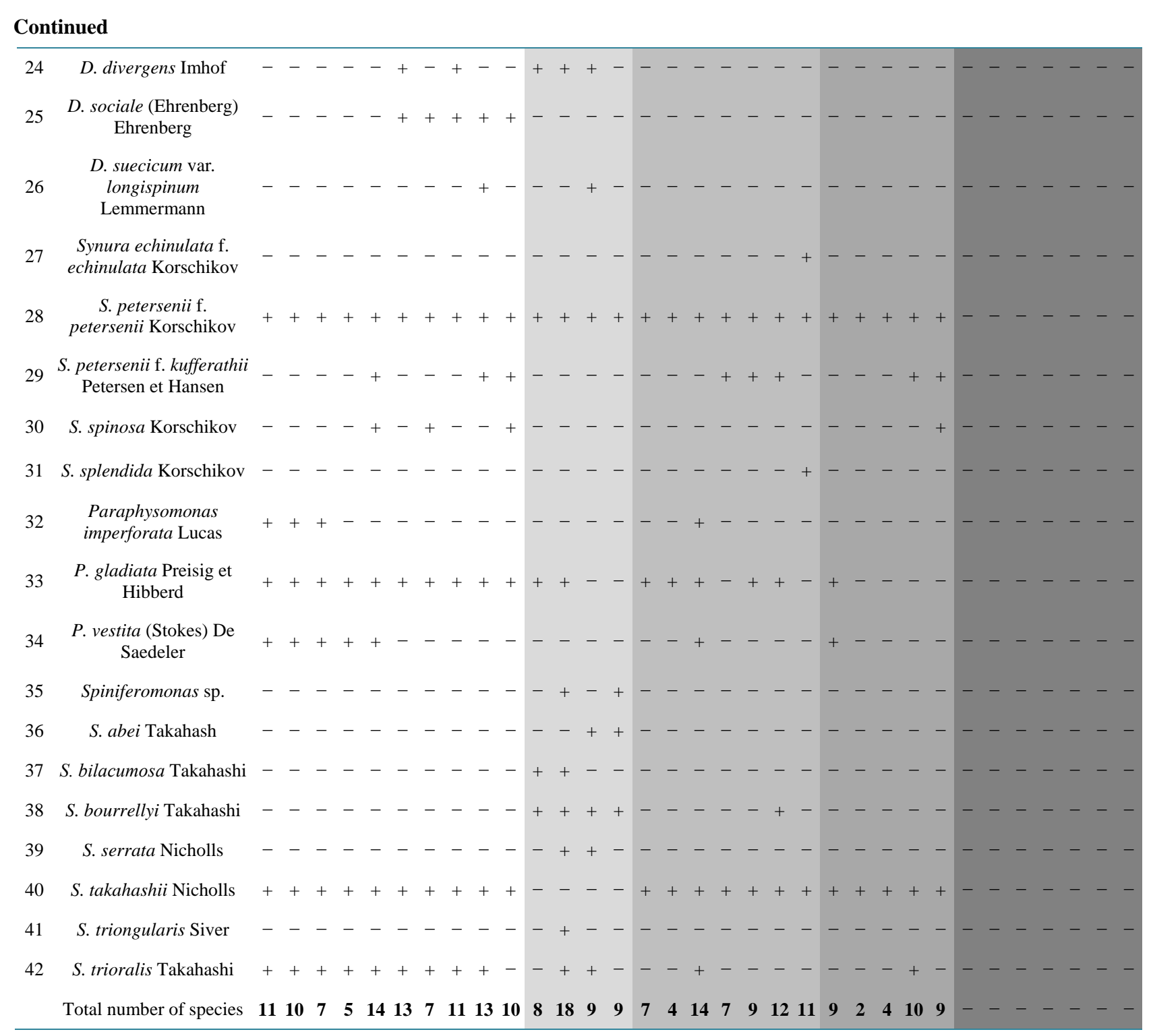

in the bay (seven species) than in the lower Yenisei, Yenisei Gulf, and Kara Sea coastal waters; species of the genera Kephyrion and Pseudokephyrion were also regularly found in plankton samples. Species such as $M$. heterospina, M. akrokomos and M. elongata were recorded at station 18, where the species diversity of chrysophytes was the highest (Table 2, Figure 2, Figure 3).

\subsection{Mixed Waters}

The diversity of chrysophytes in the Yenisei Gulf (st. 1-4, 10, 22, 23) was slightly lower (24 species). As in the lower Yenisei, the group of most frequent species included Spiniferomonas takahashii, Synura petersenii f. petersenii, S. petersenii f. kufferathii, and, to a lesser extent, Mallomonas acaroides, M. alpina, M. crassisquama and Paraphysomonas gladiata. Some chrysophytes recorded in the gulf-Synura echinulata, S. splendida (st. 10), and Chrysosphaerella coronacircumspina (st. 23, 10)_did not occur at other stations, including those in the river segment. Only a few samples contained species such as P. vestita (st. 2), M. akrokomos (st. 1), M. heterospina (st. 3), S. bourrellyi (st. 4). Chrysophytes of the genus Dinobryon occurred mainly in fresh waters of the lower Yenisei and Gydan Bay, with only single D. cylindricum cells occurring in samples of mixed waters from the Yenisei Gulf (Table 2, Figure 2, Figure 3). 

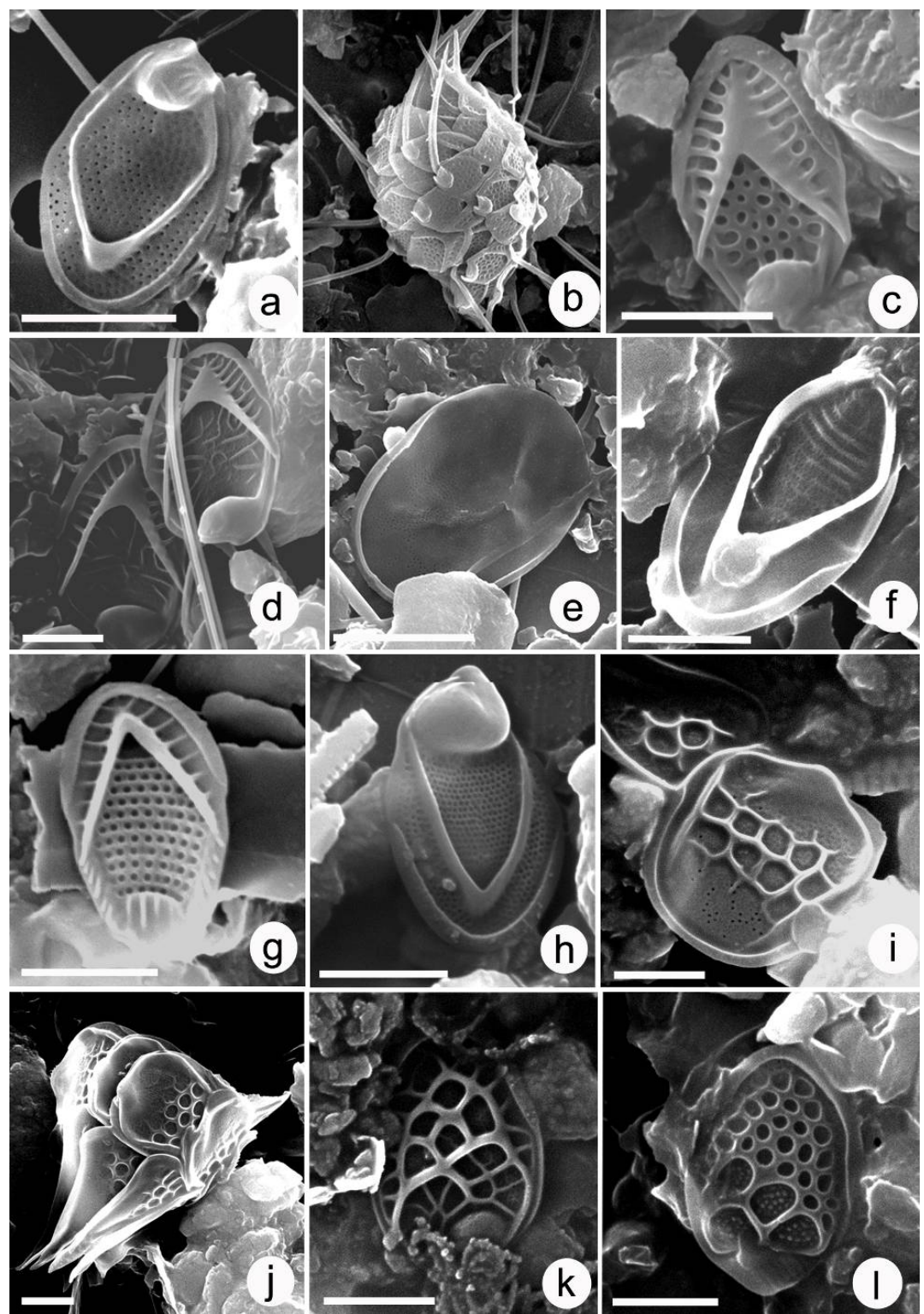

Figure 2. Scales of chrysophytes of the genus Mallomonas (SEM): (a) M. alpina, scale; (b) M. crassisquama cell and (c) scale; (d) M. acaroids, scale; (e) M. caudate, scale; (f) $M$. costata, scale; (g) M. striata var. Striata, scale; (h) M. tonsurata, scale; (i) M. punctifera, scale; and (j) cell armour; (k) M. heterospina, scale; (l) $M$. multiunca var. Pocosinensis, scale.

Samples from stations in the Kara Sea shelf (st. 5-9, 20, 21) contained a total of 19 chrysophyte species, most of them widespread and cosmopolitan (Synura petersenii f. petersenii, S. petersenii f. kufferathii, Mallomonas crassisquama, etc.). In addition, a rare form such as M. multiunca var. pocosiensis was recorded there (Table 2, Figure 2, Figure 3).

\subsection{Influence of Physicochemical Factors}

As follows in Table 2, the species diversity of chrysophytes in the study region was dependent mostly on water salinity: it reached a maximum of 19 species at st. 18 in Gydan Bay, where salinity was the lowest $(0.03 \%)$, and remained fairly high (12 species) at st. 4 in the Yenisei Gulf (3.8\% salinity), but markedly decreased in the Kara Sea shelf (st. 5-8, 9), along with increase in salinity and decrease in dissolved oxygen level. Our previous data 

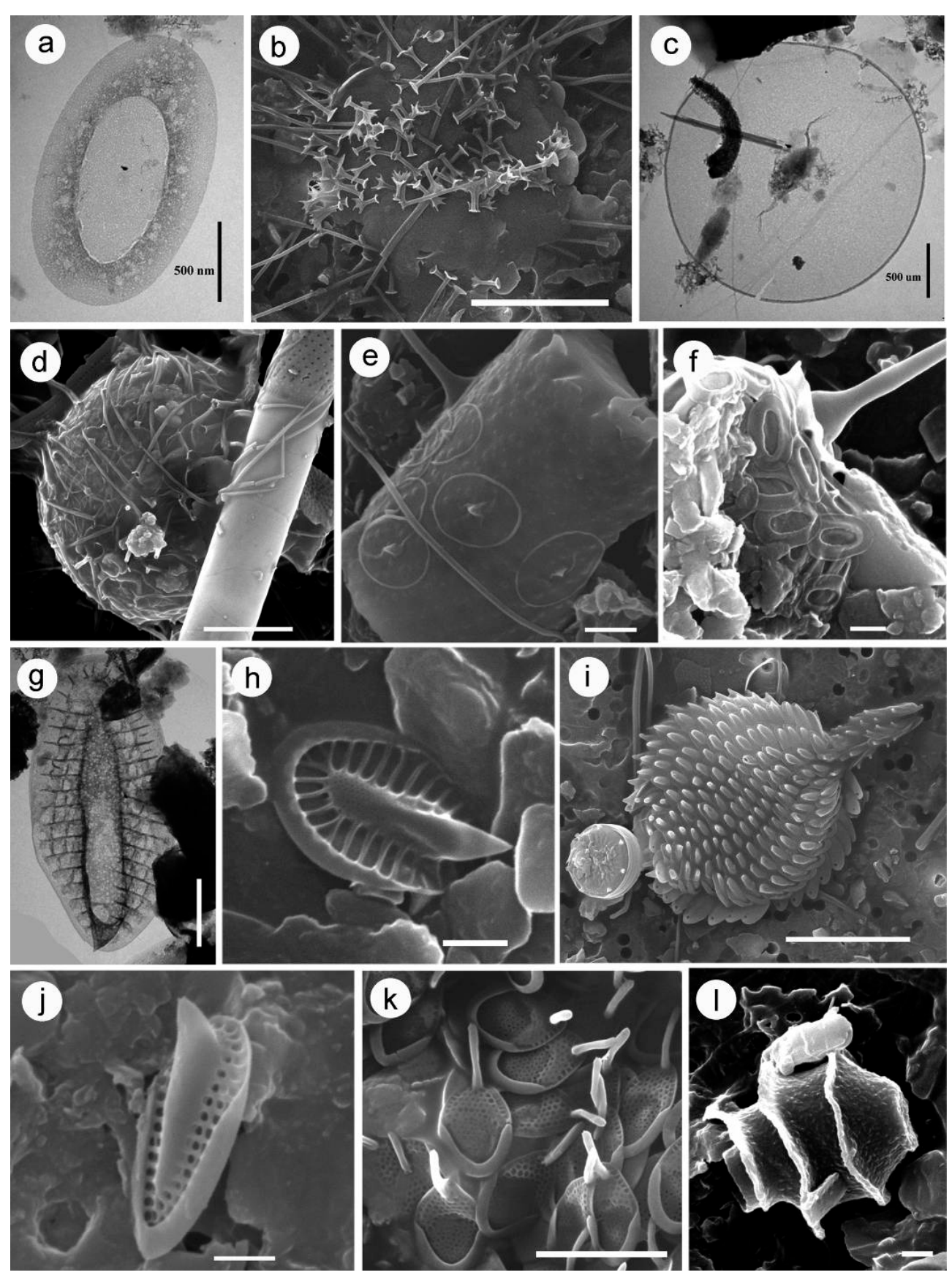

Figure 3. (a) Spiniferomonas trioralis, plate scale; (b) S. takahashii, cell armour and spine scales; (c) Paraphysomonas vestita, plate scale; (d) $P$. vestita cell with plate and spine scales; (e) $P$. gladiata, plate and spine scales; (f) Chrysosphaerella coronacircumspina, a plate scale and the base of a spine scale; (g, h) scales of Synura petersenii f. petersenii; (i) S. petersenii cell armour; (j) S. petersenii f. kufferathii scale; (k) S. spinosa, a fragment of lorica; (l) Kephyrion spirale lorica. (a, c, g) TEM; (b, d-f, h-l) SEM.

[27] show that facultative marine diatoms of the genus Thalassiosira begin to prevail in the phytoplankton of this region. Samples from st. 22 with water salinity was the highest (8\%), proved to contain chrysophytes such as Mallomonas crassisquama, Synura pertersenii, Paraphysomonas gladiata, and Spiniferomonas takahashii. Several chrysophyte species (Paraphysomonas imperforata, Mallomonas tonsurata, Paraphysomonas vestita and Synura petersenii) were previously found in brackish-water lakes of Greenland [7]. 
Table 3. Characteristics of geographic distribution (G) of chrysophytes (wc, widespread and cosmopolitan species; lr, species with limited distribution and rare species; a-a, arctalpine species) and their ranges of water physicochemical parameters in the zone of mixing of Yenisei River and Kara Sea.

\begin{tabular}{|c|c|c|c|c|c|c|c|c|c|c|}
\hline No. & G & Species & $\mathrm{T},{ }^{\circ} \mathrm{C}$ & $\mathrm{pH}$ & $\begin{array}{c}\mathrm{O}_{2}, \\
\mathrm{mg} \cdot \mathrm{L}^{-1}\end{array}$ & $\begin{array}{l}\text { Turbidity, } \\
\mathrm{mg} \cdot \mathrm{L}^{-1}\end{array}$ & Salinity, \%o & $\begin{array}{c}\mathrm{Si}, \\
\mu \mathrm{g} \cdot \mathrm{L}^{-1}\end{array}$ & $\begin{array}{l}\text { Conductivity, } \\
\mu \mathrm{S} \cdot \mathrm{cm}^{-1}\end{array}$ & $\begin{array}{c}\mathrm{P}, \\
\mu \mathrm{g} \cdot \mathrm{L}^{-1}\end{array}$ \\
\hline 1 & WC & $\begin{array}{c}\text { Chrysosphaerella } \\
\text { brevispina }\end{array}$ & $8.7-11.6$ & $7.57-8.2$ & $10.4-11.7$ & $4.34-71.5$ & $0.03-3.8$ & $0.11-3.97$ & $42.8-7410$ & $0-13$ \\
\hline 2 & WC & C. coronacircumspina & $8.4-10.4$ & $7.57-8.07$ & $10.7-11.4$ & $7.36-128.68$ & $0.03-5.2$ & $0.11-2.84$ & $42.8-7410$ & $0-26$ \\
\hline 3 & - & Kephyrion amphorula & $8.7-10.4$ & $7.57-8.06$ & $10.7-11.1$ & $14.53-71.51$ & $0.03-3.12$ & $0.11-2.67$ & $42.8-6020$ & $1-10$ \\
\hline 4 & - & K. boreale & 10.4 & 7.57 & 11.4 & 71.51 & 0.03 & 0.11 & 42.8 & 1 \\
\hline 5 & - & K. inconstans & 10.4 & 7.57 & 14.7 & 71.51 & 0.03 & 0.11 & 42.8 & 1 \\
\hline 6 & - & K. spirale & $8.4-10.8$ & $7.57-8.06$ & $10.85-14.7$ & $7.74-128.68$ & $0.03-5.2$ & $0.11-2.84$ & $42.8-546$ & $0-26$ \\
\hline 7 & WC & Mallomonas acaroides & $8.7-11.6$ & $7.95-8.2$ & $9.7-11.7$ & $4.34-68.87$ & $0.1-3.8$ & $2.05-3.97$ & $158-7150$ & $2-15$ \\
\hline 9 & WC & M. akrokomos & $8.2-11.6$ & $7.57-8.09$ & $8.7-11.1$ & $5.85-71.51$ & $0.03-2.5$ & $0.11-3.97$ & $215-5180$ & $1-11$ \\
\hline 10 & wC & M. caudata & $8.7-11.6$ & $7.95-8.09$ & $10.4-10.9$ & $5.85-27.17$ & $0.1-3.8$ & $2.05-3.97$ & $158-7410$ & $6-13$ \\
\hline 11 & - & M. costata & 9.9 & 8.04 & 10.7 & 17.36 & 0.2 & 2.26 & 299 & 13 \\
\hline 12 & WC & M. crassisquama & $8.1-11.6$ & $7.57-8.11$ & $10.4-11.1$ & $4.34-128.68$ & $0.03-8$ & $0.11-3.97$ & $42.8-12,730$ & $1-15$ \\
\hline 13 & $\operatorname{lr}$ & M. elongata & 10.4 & 7.57 & 11.1 & 71.51 & 0.03 & 0.11 & 42.8 & 1 \\
\hline 14 & WC & M. heterospina & $9.5-10.3$ & $7.64-7.98$ & $10.7-10.9$ & $13.02-85.27$ & $0.04-3.12$ & $0.15-3.03$ & $86.8-5540$ & $2-9$ \\
\hline 15 & $\operatorname{lr}$ & $\begin{array}{l}\text { M. multiunca var. } \\
\text { pocosinensis }\end{array}$ & $9.9-10.8$ & $7.93-8.11$ & 10.9 & 7.74 & $0.1-3$ & $2.01-3.03$ & $161.3-6060$ & $6-12$ \\
\hline 16 & wC & M. punctifera & $8.7-11.6$ & $7.95-8.11$ & $10.4-10.9$ & $6.04-14.53$ & $0.1-8$ & $0.02-3.97$ & $158-7410$ & $6-13$ \\
\hline 18 & WC & M. tonsurata & $9.5-11.6$ & $7.95-8.09$ & $10.4-11.7$ & $4.34-14.53$ & $0.1-1$ & $2.05-3.97$ & $158-2000$ & $2-8$ \\
\hline 19 & - & Pseudokephyrion entzii & 10.6 & 7.55 & 11.3 & 197.55 & 0.1 & 0.11 & 205 & 5 \\
\hline 20 & - & P. undulatissimum & 10.6 & 7.55 & 11.3 & 197.55 & 0.1 & 0.11 & 205 & 5 \\
\hline 21 & - & Chrysococcus furcatus & 9.6 & 7.65 & 11.3 & 60 & 0.2 & 0.14 & 546 & 0 \\
\hline 22 & $\mathrm{a}-\mathrm{a}$ & Dinobryon bavaricum & 9.2 & 7.95 & 11.0 & 68.87 & 0.4 & 2.02 & 704 & 15 \\
\hline 23 & WC & D. cylindricum & $8.4-9.5$ & $7.95-7.98$ & $10.8-11.0$ & $14.53-128.68$ & $0.14-5.2$ & $2.05-2.84$ & $158-9030$ & $8-26$ \\
\hline 24 & WC & D. divergens & $9.2-10.6$ & $7.55-7.95$ & $10.3-11.0$ & $14.53-197.55$ & $0.003-0.4$ & $0.11-2.21$ & $42.8-704$ & $1-15$ \\
\hline 25 & wC & D. sociale & $8.8-9.9$ & $7.95-8.04$ & $10.7-11.1$ & $14.53-68.87$ & $0.14-0.2$ & $2.02-2.29$ & $158-704$ & $6-15$ \\
\hline 26 & - & $\begin{array}{l}\text { D. suecicum var. } \\
\text { longispinum }\end{array}$ & $9.8-10.3$ & $7.64-8.04$ & 10.9 & $27.14-85.28$ & $0.04-0.16$ & $0.15-2.25$ & $86.8-215$ & $2-11$ \\
\hline 27 & WC & $\begin{array}{c}\text { Synura echinulata f. } \\
\text { echinulata }\end{array}$ & 9.6 & 8.01 & 10.7 & 12.83 & 3.12 & 0.92 & 6020 & 10 \\
\hline 28 & WC & S. petersenii Korshikov & $8.1-10.8$ & $7.55-8.11$ & $8.7-11.7$ & $4.34-197.55$ & $0.03-8$ & $0.11-3.97$ & $42.8-12,730$ & $2-26$ \\
\hline 29 & $\operatorname{lr}$ & S. petersenii f. kufferathii & $8.7-10.2$ & $7.98-8.11$ & $10.7-10.9$ & $6.04-27.14$ & $0.1-3.8$ & $2.09-3.31$ & $163-7150$ & $6-13$ \\
\hline 30 & wC & S. spinosa & $8.7-9.9$ & $7.96-8.11$ & $10.7-11.1$ & $14.53-20.57$ & $0.1-3$ & $2.02-3.03$ & $163-6060$ & $6-8$ \\
\hline 31 & - & S. splendida & 9.6 & 8.01 & 10.7 & 12.83 & 3.12 & 2.67 & 6020 & 10 \\
\hline
\end{tabular}




\begin{tabular}{|c|c|c|c|c|c|c|c|c|c|c|}
\hline \multicolumn{11}{|c|}{ Continued } \\
\hline 32 & wc & $\begin{array}{l}\text { Paraphysomonas } \\
\text { imperforata }\end{array}$ & $10.2-11.6$ & $7.93-8.02$ & $10.4-11.7$ & $4.34-8.11$ & $0.1-1$ & $2.01-3.97$ & $161.3-2000$ & $2-8$ \\
\hline 33 & $\mathrm{lr}$ & P. gladiata & $8.1-11.6$ & $7.55-8.2$ & $10.4-11.7$ & $4.34-128.68$ & $0.03-8$ & $0.11-3.97$ & $42.8-12,730$ & $1-26$ \\
\hline 34 & wc & P. vestita & $8.7-10.8$ & $7.93-8.2$ & $10.4-11.7$ & $4.34-14.53$ & $0.1-1$ & $1.97-3.97$ & $158-6050$ & $2-9$ \\
\hline 35 & - & Spiniferomonas sp. & $9.6-10.4$ & $7.57-7.95$ & $11.1-11.3$ & $60.0-71.51$ & $0.03-0.2$ & $0.11-0.14$ & $42.8-546$ & $0-1$ \\
\hline 36 & - & S. $a b e i$ & $9.6-10.3$ & $7.64-7.65$ & $10.9-11.3$ & $60.0-85.28$ & $0.04-0.2$ & $0.14-0.15$ & $86.8-546$ & $0-2$ \\
\hline 37 & wc & S. bilacumosa & $10.4-10.6$ & $7.55-7.57$ & $11.1-11.3$ & $71.51-197.55$ & $0.03-0.1$ & 0.11 & $42.8-205$ & $1-5$ \\
\hline 38 & wc & S. bourrellyi & $9.6-10.6$ & $7.55-8.03$ & $10.7-11.3$ & $6.04-197.55$ & $0.03-3.8$ & $0.11-2.85$ & $42.8-7150$ & $0-6$ \\
\hline 39 & - & S. serrata & $10.3-10.4$ & $7.57-7.64$ & $10.6-11.1$ & $71.51-85.87$ & $0.03-0.04$ & $0.11-0.15$ & $42.8-86.8$ & $1-2$ \\
\hline 40 & - & S. takahashii & $8.2-11.6$ & $7.95-8.11$ & $8.6-11.7$ & $4.34-128.68$ & $0.1-5.2$ & $1.97-3.97$ & $158-12,730$ & $2-26$ \\
\hline 41 & - & S. triangularis & 10.4 & 5.57 & 11.1 & 71.51 & 0.03 & 0.11 & 42.8 & 1 \\
\hline 42 & - & S. trioralis & 8.7 - 11.6 & $7.57-8.2$ & $10.4-11.7$ & $4.34-85.28$ & $0.03-3.8$ & $0.11-3.97$ & $158-7410$ & $1-15$ \\
\hline
\end{tabular}

Most chrysophytes concentrated in water layers rich in oxygen. Their diversity was the highest at st. 18, where the dissolved oxygen level reached $11.1 \mathrm{mg} \cdot \mathrm{l}^{-1}$, but sharply decreased at st. 7 , where this level was low. It is noteworthy that samples from the latter station contained species such as Mallomonas akrokomos, M. striata, Synura petersenii and Spiniferomonas takahashii.

Water $\mathrm{pH}$ is an important factor governing the distribution of chrysophytes, and its role has been well studied. As shown previously, the optimum for the development of these algae in water bodies of northern Russia is at pH 5.5 - 7.5 [14], while the number of chrysophyte species in the tributaries of Lake Ladoga in the springsummer season reaches a peak at pH 7.5 - 7.92 [26]. We found that the diversity of chrysophytes was maximum at st. 18 in Gydan Bay, where water $\mathrm{pH}$ was 7.57, and decreased in waters with higher $\mathrm{pH}$ values. The literature provides detailed data on the response to $\mathrm{pH}$ of species from the genera Mallomonas and Synura [1] [28]-[30]. In particular, M. akrokomos and $M$. caudata are tolerant of wide $\mathrm{pH}$ range (4.0 - 9.0 and 4.5 - 9.0, respectively), and the same is true of Synura petersenii ( $\mathrm{pH} 3.0$ - 9.0). In the region of our study, the development of these species reached a peak at pH 7.57 - 8.09 and 7.95 - 8.09, respectively. It is considered that Mallomonas acaroides in nature prefers alkaline waters with $\mathrm{pH}>8.0$ [28] [29]. Our study confirmed the occurrence of this species in samples from waters with $\mathrm{pH}$ 8.2, which also contained Chrysosphaerella brevispina, Mallomonas alpina, Paraphysomonas gladiata, $P$. vestita and Spiniferomonas trioralis. At the lowest $\mathrm{pH}$ (7.55), species such as $M$. striata was recorded (Table 3).

It is known that even slight water turbidity leads to reduction of the species composition of planktopnic algae [31]. According to our data, however, this factor had no significant effect on the diversity of chrysophytes, except for the situation at st. 19 in Gydan Bay: water turbidity at this station was increased, and only eight chrysophyte species were recorded there, compared to a maximum of 19 species at the neighboring st. 18 (Table 1, Table 2).

An increase in the concentration of mineral phosphorus and consequent eutrophication of water bodies lead to reduction of chrysophyte species diversity. Species prevailing in waters with high phosphorus concentrations included Chrysosphaerella coronacircumspina, Kephyrion spirale, Dinobryon cylindricum, Synura petersenii, Paraphysomonas gladiata and Spiniferomonas takahashii (Table 3). Fluctuations of water temperature were minor and had no significant effect on chrysophyte species diversity in the study region.

Synura petersenii and Spiniferomonas takahashii, the most abundant and frequent species in the lower Yenisei and Yenisei Gulf, proved to successfully develop in a wide range of ecological (environmental) factors (Table 2).

Appropriate habitat conditions were also determined for rare species such as M. elongata (st. 18), M. multiunca var. pocosiensis (st. 9, 33), S. petersenii f. kufferathii (st. 2-4, 8, 9, 24, 25, 30), and P. gladiata (st. 1, 3, 4, 5, 18, 19, 22-34) (Table 3). Mallomonas elongata occurred at st. 18, in waters with the lowest salinity and pH; M. multiunca var. pocosiensis, at two stations in the lower Yenisei (st. 33) and Kara Sea shelf (st. 9) where water 
pH was the highest; and S. petersenii f. kufferathii, at 18 stations with increased water $\mathrm{pH}$ and salinity. According to Voloshko et al. [26], S. petersenii f. kufferathii in Lake Ladoga successfully developed at water $\mathrm{pH}$ of 6.75 to 7.31 and mineral phosphorus content of $15-85 \mu \mathrm{g} \cdot \mathrm{l}^{-1}$, whereas the upper limit of $\mathrm{pH}$ range for this species in the study region extended to $\mathrm{pH}$ 8.11, mineral phosphorus content of $6-13 \mu \mathrm{g} \cdot \mathrm{l}^{-1}$. Paraphysomonas gladiata in our study was recorded at 17 stations, in a wide range of salinity and $\mathrm{pH}$, and showed preference for waters rich in dissolved oxygen. Its development in Lake Ladoga was observed at $\mathrm{pH} 7.18$ - 7.92 and 0 - 19 $\mu \mathrm{g} \cdot \mathrm{l}^{-1}$ of mineral phosphorus [26], but our data show that this species occurs in waters with higher values of these parameters (Table 3 ).

\subsection{Comparison of Species Composition}

Comparisons of chrysophyte species composition between sampling stations showed that representatives of the genus Mallomonas were the most diverse group of these algae (Figure 4), as was also observed in other Arctic regions [4] [7] [11] [12] [17]. This genus comprises boreal and cosmopolitan species, most of them being oligosaprobiontic and indifferent to water salinity. Species of the genera Dinobryon and Kephyrion prevailed in fresh waters, at the mouths of tributaries flowing into the lower Yenisei and at coastal stations in Gydan Bay (Figure 4).

An analysis of chrysophyte species composition between the study region and some other regions with similar climatic conditions revealed 81\% similarity (Sørensen's coefficient) of chrysophyte flora in the lower Yenisei to that in some water bodies of the Taimyr Peninsula [8].

\section{Conclusions}

Analysis of phytoplankton samples from the study region by electron microscopic methods (SEM and TEM) allowed us to identify scaled chrysophytes from nine genera-Mallomonas, Spiniferomonas, Chrysosphaerella, Paraphysomonas, Synura, Dinobryon, Pseudokephyrion, Kephyrion, and Chrysococcus-and thereby expanded the species list of these algae in the Arctic zone. Some rare representatives of Chrysophyta were discovered in the samples, such as Synura petersenii f. kufferathii and Paraphysomonas gladiata previously found in lakes of

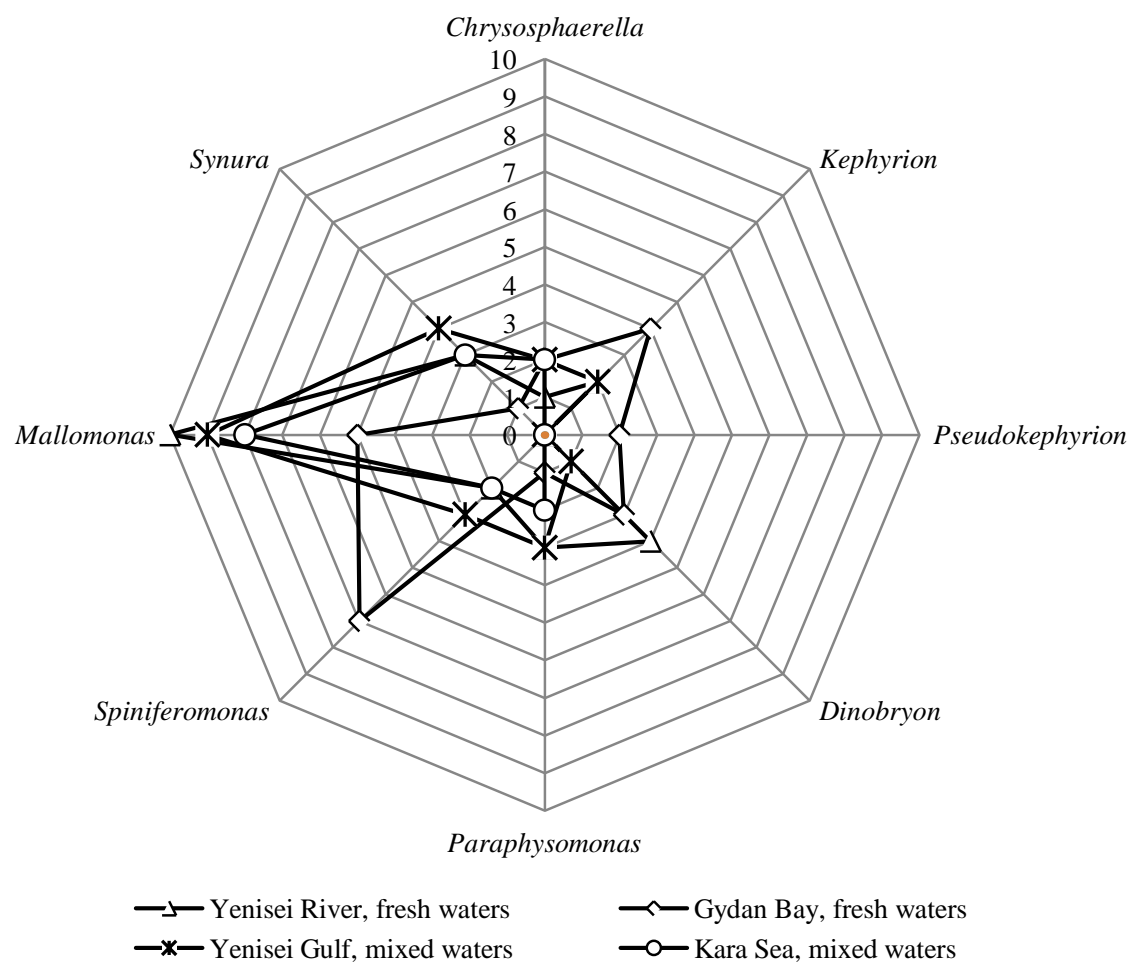

Figure 4. Changes in chrysophyte species composition depending on water salinity in the study region. 
Bol'shezemel'skaya tundra [11] and Taimyr Peninula [8] and Mallomonas multiunca var. pocosiensis described from Northern California [32].

The taxonomic composition of Chrysophyta in the study region was similar to that in some water bodies of the Taimyr Peninsula. Their species diversity depends on water salinity, being higher in fresh or freshened waters. A major proportion of chrysophytes in the region (more than 10 species) inhabit water with the following parameters: salinity $0.03 \%$ - 3.8\%o, $\mathrm{pH} 7.57-8.06$, turbidity $5.85-71.54 \mathrm{mg} \cdot \mathrm{l}^{-1}$, mineral phosphorus $0-0.013$ $\mathrm{mg} \cdot \mathrm{l}^{-1}$, dissolved oxygen $10.4-11.7 \mathrm{mg} \cdot \mathrm{l}^{-1}$. Synura petersenii and Spiniferomonas takahashii were proved to have the widest ecological range.

\section{Acknowledgements}

We are grateful to G. I. Popovskaya, N. A. Bondarenko, and L. N. Voloshko for their valuable comments on the manuscript.

This study was performed using the facilities of the Instrumentation Center for Electron Microscopy at the Integrated Center of Ultramicroanalysis (Limnological Institute, Siberian Branch, Russian Academy of Sciences). Expedition research and hydrochemical analysis were supported by "Complex Researches of the Arctic Shelf" the Presidium of the Russian Academy of Sciences (project No. 23.6). Analysis of chrysophytes was performed according to the budged-funded program of the Limnological Institute (project No. VI. 50.1.3).

\section{References}

[1] Kristiansen, J. (2005) Golden Algae: A Biology of Chrysophytes. A.R.G. Gantner Verlag, Königstein, 167 p.

[2] Matvienko, A.M. (1954) Zolotistye vodorosli. Opredelitel’ presnovodnykh vodoroslei SSSR (Chrysophyte Algae: Identification Key to Freshwater Algae of the Soviet Union). Sovetskaya Nauka, Moscow, 188 p.

[3] Starmach, K. (1985) Chrysophyceae und Haptophyceae. VEB Gustav Fischer Verlag, Jena, 515 p.

[4] Kristiansen, J. and Preisig, H.R. (2007) Chrysophyte and Haptophyte Algae. 2 Teil/Part 2: Synurophyceae. In: Budel, B., Gartner, G., Krienitz, L., Preisig, H.R. and Schagerl, M., Eds., Freshwater Flora of Central Europe, Springer Verlag, Berlin Heidelberg, 252 p.

[5] Sandgren, C.D., Smol, J.P. and Kristiansen, J. (1995) Chrysophyte Algae: Ecology, Phylogeny and Development. Cambridge University Press, New York, 384 p. http://dx.doi.org/10.1017/CBO9780511752292

[6] Zeeb, B.A. and Smol, J.P. (2001) Chrysophyte Scales and Cysts. Vol. 3, In: Smol, J.P., Birks, H.J.B. and Last, W.M., Eds., Tracking Environmental Change Using Lake Sediments, Kluwer Academic Publishers, Dordrecht, 203-223.

[7] Kristiansen, J. (1992) Silica-Scaled Chrysophytes from West Greenland: Disko Island and the Søndre Strømfjord Region. Nordic Journal of Botany, 12, 525-536. http://dx.doi.org/10.1111/j.1756-1051.1992.tb01831.x

[8] Kristiansen, J., Dowel, L. and Wegeberg, S. (1997) Silica-Scaled Chrysophytes from the Taymyr Peninsula, Northern Siberia. Nova Hedwigia, 65, 337-351.

[9] Laybourn-Parry, J. and Marshall, W.A. (2003) Photosynthesis, Mixotrophy and Microbial Plankton Dynamics in Two High Arctic Lakes during Summer. Polar Biology, 26, 517-524. http://dx.doi.org/10.1007/s00300-003-0514-z

[10] Douglas, M.S.V., Smol, J.P. and Blake Jr., W. (1994) Marked Post-18th Century Environmental Change in High-Arctic Ecosystems. Science, 266, 416-419. http://dx.doi.org/10.1126/science.266.5184.416

[11] Siver, P.A., Voloshko, L.N., Gavrilova, O.V. and Getsen, M.V. (2005) The Scaled Chrysophyte Flora of the Bolshezemelskaya Tundra (Russia). Nova Hedwigia, 128, 125-150.

[12] Voloshko, L.N. (2007) Chrysophyte Flora from Glacial Lakes in the Kara River Basin, the Polar Urals. Proceedings of the V European Congress of Protistology, St. Petersburg, 23-27 July 2007, 127-128.

[13] Voloshko, L.N. (2011) Chrysophyte Algae in the Ecosystem of Northern Latitudes: New Approaches to the Study of Biodiversity. In: Getsen, M.V., Patova, E.N., Barionova, S.S., et al., Eds., Vodorosli: Taksonomiya, ekologiya, ispol'zovanie v monitoringe (Algae: Taxonomy, Ecology, Applications in Monitoring), Ural Branch of the Russian Academy of Sciences, Yekaterinburg, 22-28.

[14] Voloshko, L.N. (2012) A New Species of the Genus Mallomonas (Chrysophyta, Synurophyceae) from Lakes of the Vorkuta Tundra. Botanicheskii Zhurnal, 97, 1090-1098.

[15] Voloshko, L.N. (2012) Chrysophyte Algae (Chrysophyceae, Synurophyceae) in Water Bodies of Northern Russia. Extended Abstract of Doctoral Dissertation, Saint Petersburg State University, St. Petersburg, 43 p.

[16] Rautio, M., Dufresne, F., Laurion, I., Bonilla, S., Vincent, W.F. and Christoffersen, K.S. (2011) Shallow Freshwater Ecosystems of the Circumpolar Arctic. Écoscience, 18, 204-222. http://dx.doi.org/10.2980/18-3-3463 
[17] Pichrtová, M., Janatková, K. and Němcová, Y. (2011) Silica-Scaled Chrysophytes from Abisko (Swedish Lapland). Nordic Journal of Botany, 29, 112-118. http://dx.doi.org/10.1111/j.1756-1051.2010.00809.x

[18] Charvet, S., Vincent, W.F. and Lovejoy, C. (2012) Chrysophytes and Other Protists in High Arctic Lakes: Molecular Gene Surveys, Pigment Signatures and Microscopy. Polar Biology, 35, 733-748. http://dx.doi.org/10.1007/s00300-011-1118-7

[19] Voloshko, L.N., Getsen, M.V. and Gavrilova, O.V. (2005) Chrysophyte Algae in the Ecosystem of Northern Latitudes. In: Genkal, S.I., Ed., Morphologiya, sistematika, ontogenez, ekologiya i biogeografiya diatomovykh vodoroslei (Morphology, Systematics, Ontogeny, Ecology, and Biogeography of Diatoms), Institute of Inland Water Biology, Russian Academy of Sciences, Borok, Russia, 30.

[20] Voloshko, L.N. (2009) New Taxa of the Genus Mallomonas (Chrysophyta, Synurophyceae) from Lakes of the Polar Urals. Botanicheskii Zhurnal, 94, 1068-1076.

[21] Voloshko, L.N. (2010) The Chrysophycean Algae from Glacial Lakes of Polar Ural (Russia). Nova Hedwigia, 136, 191-121.

[22] Balonov, I.M. and Kuz’mina, A.E. (1986) Chrysophyte Algae. In: Votintsev, K.K., Ed., Gidrokhimicheskie i gidrobiologicheskie issledivaniya Khantaiskogo vodokhranilishcha (Hydrochemical and Hydrobiological Studies in the Khantay Reservoir), Trudy Limnologicheskogo Instituta Sibirskogo Otdeleniya Akademii Nauk SSSR, Nauka, Novosibirsk, 59-70.

[23] Wetzel, R.G. and Likens, G.E. (1991) Limnological Analyses. Springer-Verlag, New York. http://dx.doi.org/10.1007/978-1-4757-4098-1

[24] Bordovsky, O.K. and Ivanenkov, V.N., Eds. (1978) Metody gidrokhimicheskikh issledovanii okeana (Methods for Hydrochemical Studies in the Ocean). Nauka, Moscow, 271 p.

[25] Baram, G.I., Vereshchagin, A.L. and Golobokova, L.P. (1999) Application of Microcolumn HPLC with UV Detection to Analysis of Anions in Environmental Objects. Zhurnal Analiticheskoi Khimii, 54, 962-965.

[26] Voloshko, L.N., Gavrilova, O.V. and Gromov, B.V. (2002) The Diversity of Chrysophyte Algae (Paraphysomonadaceae, Mallomonadaceae, Synuraceae) in Lake Ladoga and Its Region. Al'gologiya, 12, 25-35.

[27] Bessudova, A.Y., Sorokovikova, L.M., Firsova, A.D., Tomberg, I.V. and Likhoshway, Y.V. (2014) Changes in Phytoplankton Community Composition along a Salinity Gradient from the Lower Yenisei River to the Kara Sea, Russia. Botanica Marina, 57, 225-239. http://dx.doi.org/10.1515/bot-2013-0102

[28] Siver, P.A. (1989) The Separation of Mallomonas acaroides v. acaroides and v. muskokana (Synurophyceae) along a pH Gradient. Nova Hedwigia, 95, 111-117.

[29] Siver, P.A. (1991) The Stomatocyst of Mallomonas acaroides v. muskokana (Chrysophyceae). Journal of Paleolimnology, 5, 11-17. http://dx.doi.org/10.1007/BF00226556

[30] http://www.chrysophytes.eu

[31] Sudnitsina, D.N. (2005) Ekologiya vodoroslei Pskovskoi oblasti. Uchebnoe posobie (The Ecology of Algae in Pskov Oblast: A Textbook). Pskov State Polytechnic Institute, Pskov, 128 p.

[32] Siver, P.A. (2003) Mallomonas multiunca var. pocosinensis var. nov. (Synurophyceae) from Freshwater Localities along Coastal Sections of North Carolina, USA. Nova Hedwigia, 76, 147-156. http://dx.doi.org/10.1127/0029-5035/2003/0076-0147. 